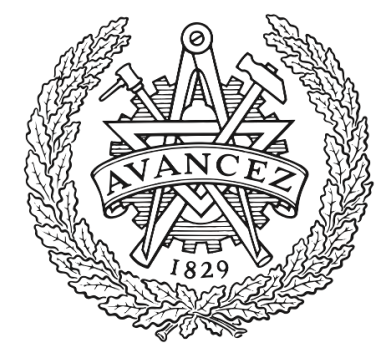

CHALMERS

UNIVERSITY OF TECHNOLOGY

\title{
Transient heat transfer and moisture load in cold attic constructions - $A$ CFD analysis
}

Downloaded from: https://research.chalmers.se, 2023-04-26 09:21 UTC

Citation for the original published paper (version of record):

Shankar, V., Hellsvik, R., Hagentoft, C. (2018). Transient heat transfer and moisture load in cold attic constructions - A CFD analysis. AIP Conference Proceedings, 1978.

http://dx.doi.org/10.1063/1.5044081

N.B. When citing this work, cite the original published paper. 


\title{
Transient Heat Transfer and Moisture Load in Cold Attic Constructions - A CFD Analysis
}

\author{
Vijay Shankar ${ }^{1, a)}$ Ragnar Hellsvik ${ }^{2}$ and Carl-Eric Hagentoft ${ }^{3}$ \\ ${ }^{1}$ Lulea University of Technology, Sweden \\ ${ }^{2}$ Chalmers University of Technology, Sweden. \\ ${ }^{3}$ Professor in Building Physics, Chalmers University of Technology, Sweden. \\ a) Corresponding author: vijay.shankar@1tu.se
}

\begin{abstract}
Mold growth in cold attic constructions has become an increasing problem in Sweden and other countries with cold climates due to the demands on energy efficient building envelopes. Highly insulated building envelopes with cold ventilated attics lead to colder climates in the attic space, which increases the risk for mold growth. In this paper, the transient heat transfer process including natural and forced convection is investigated for a 24 -hour cycle in a virtual 3D model of a ventilated attic construction by the use of CFD technology. The momentum and energy equations along with the realizable k-epsilon turbulence model are solved with commercial CFD software. Transient simulations are performed for daily temperature variations for various ventilation rates and a hygrothermal analysis is conducted to estimate the risk for mold growth at the inner roof boundary. A virtual model of a ventilated attic with an underlying layer of porous medium is used for the simulations and the CFD approach is based on a validated model from previous research. The results are the temperature field and the risk for mold growth for different ventilation rates presented as a function of time during the cycle.
\end{abstract}

\section{Keywords}

cold attics, computational fluid dynamics (CFD), heat transfer, moisture load, building physics, fluid mechanics

\section{INTRODUCTION}

In the southern and west part of Sweden, building envelopes that are built or retrofitted with thick insulation layers, are susceptible to mold growth a problem that has increased remarkably over the last decade [1]. The correlation between the local climate and ventilation rates, with the buildup of moisture is of great interest and recent research [2] shows that greatly reduced, or completely eliminated risk for mold growth can be achieved by controlling the ventilation rate. By investigating the heat transfer process and the flow pattern numerically, knowledge and understanding about their correlation can be obtained in a more efficient way than by performing field measurements. For this investigation the commercial CFD software ANSYS Fluent has been used.

The ventilation in cold attics is usually driven by wind, which forces air into the attic through a gap at one of the eaves (see Figure 1). The ventilated air can then exit the attic through another gap at the opposite side or through an outlet at the top of the roof. The chosen configuration of the ventilation for the attic model is presented in Figure 1 , which shows the cross section of the geometry. The attic model is $1800 \mathrm{~mm}$ deep in the direction normal to the cross section in Figure 1. The thickest part of the insulation is $400 \mathrm{~mm}$ and the slope of the roof is 20 degrees. Within the insulation lies wooden joists with spacing of $600 \mathrm{~mm}$, a width of $45 \mathrm{~mm}$ and a height of $135 \mathrm{~mm}$, shown in Figure 2. The presence of joists has a significant impact on the heat transfer as well as the flow pattern in the insulation layer [3]. 
Extensive literature study has shown that the previous research performed so far has only dealt with certain aspects $[3,4,5,6,7,8,9,10,11,12]$. This CFD attic model was initially developed by the authors in a previous research $[10,11,12]$ where it was validated by comparison with experimental work performed by [13].

Data files with hourly measured temperature data from weather stations in Sweden were analyzed and a 24-hourinterval in the middle of April was used to set the external Dirichlet boundary conditions for the external air of the attic. Simulations were performed with transient temperatures, $T_{\text {amb }}$ and $T_{\text {eq }}$, for the ventilated air and the roof boundary respectively, where $T_{\text {amb }}$ denotes ambient temperature and $T_{\mathrm{eq}}$ denotes equivalent temperature which includes effects of solar radiation, long wave radiation, convection and transfer of latent heat. The bottom of the attic floor insulation was set to a constant temperature, $\mathrm{T}_{\text {hot }}$, of $294 \mathrm{~K}$. Three transient simulations where performed for three, constant, inlet velocities corresponding to two, four and eight complete air displacements per hour. The ventilation rates for attics are usually in the range of 0-10 complete air displacements per hour.

The computational mesh was built with hexahedronal elements in the commercial software ANSYS ICEM and a mesh independence study was performed for mesh sizes from 148000 to 1125000 computational cells showing that a mesh size of approximately 500000 cells was sufficient. The Realizable k- $\varepsilon$ model was used to model turbulence in the air cavity while the insulation was modeled as a laminar zone.

\section{MATHEMATICAL FORMULATION (CFD)}

The main equations that have been solved for this problem are the governing equations for conservation of mass, momentum and energy along with the equations for the Realizable k- $\varepsilon$ model with standard wall functions [14]. The P1 model [15] has been used to predict the radiative heat transfer in the domain which solves the transport equation for radiation.
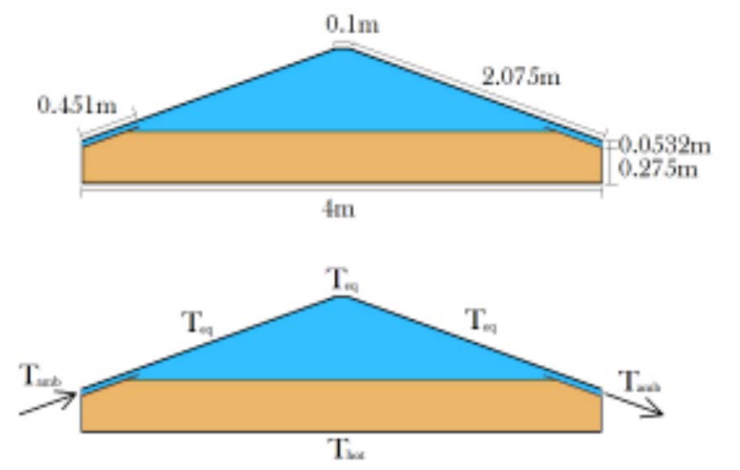

FIGURE 1. Cross section of the attic model. The upper picture gives the dimensions of the model and the lower picture shows placement of inlet and outlet as well as the temperature boundary conditions. The triangular shaped region represents the air cavity and the lower one the insulation layer.

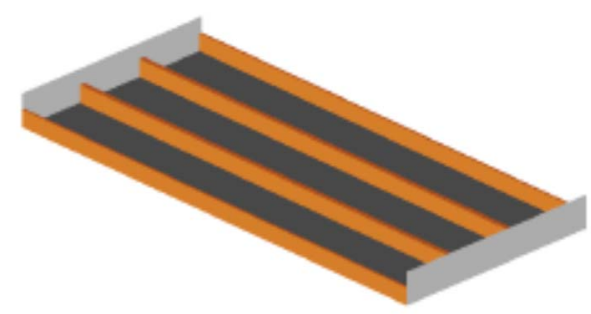

FIGURE 2. Placement of the wooden joists. 


\section{Porous Media}

The insulated volume is defined as a porous zone in which the porosity $\phi$ is defined as the fraction of the total volume that is occupied by voids [16]. The volume that is occupied by solid material in a porous medium with porosity, $\phi$, is thus $1-\phi$. The porous zone is modeled with the addition of an extra momentum source term, Equation 1, to the original transport equations consisting of the viscous and inertial losses.

$$
S_{i}=\left(\frac{\mu}{K} u_{i}+C_{2} \frac{1}{2}|u| u_{i}\right)
$$

where $\mathrm{K}\left[\mathrm{m}^{2}\right]$ is the permeability of the insulation material and $\mathrm{C}_{2}\left[\mathrm{~m}^{2} / \mathrm{kg}\right]$ is the inertial resistance factor which is usually small compared to the viscous losses for laminar flow, i.e. when the velocities are low. In such cases, the model reduces to Darcys law. $\mu\left[\mathrm{m}^{2} / \mathrm{s}\right]$ is the dynamic viscosity of the fluid, $u_{i}[\mathrm{~m} / \mathrm{s}]$ is the local velocity and $\rho\left[\mathrm{kg} / \mathrm{m}^{3}\right]$ is the density of the fluid.

\section{RELEVANT PARAMETERS}

This section briefly presents the important dimensionless parameters used in this study.

\section{Nusselt Number}

The Nusselt number is the ratio between the heat flux with and without convection. It is defined as:

$$
N u=\frac{q_{\text {conduction }}+q_{\text {convection }}}{q_{\text {conduction }}}
$$

This parameter is used in this study to describe the impact of convection on the total heat transfer process within the porous medium.

\section{Humidity and Mold Growth Potential}

The relative humidity defined in Equation 3 is used in this study to calculate a potential for the risk of mold growth according to [17]. The mold growth potential, $\mathrm{m}$, is a number between 0 and 1 , corresponding to no growth and initiation of mold growth respectively.

$$
R H=\frac{v_{a}}{v_{s}}
$$

Here, $v_{a}$ [ $\left.\mathrm{g}_{\text {water }} / \mathrm{kg}_{\text {air }}\right]$ is the humidity by volume in the attic space including contributions from ventilated air and leakages from the living area through the attic floor. The increase in the humidity level due to leakage from the living area for different ventilation rates is given in Table 1.

$v_{s}\left[\mathrm{~g}_{\text {water }} / \mathrm{kg}_{\text {air }}\right]$ is the humidity by volume at saturation approximated as:

$$
v_{S}=\frac{a *\left(b+\frac{T}{100}\right)^{n}}{461.4 \cdot(T+273.15)}
$$

TABLE 1. Additional moisture for different ventilation rates

\begin{tabular}{lll}
\hline & Ventilation rate, $\mathrm{n}$ & $v_{a}-v_{e}$ \\
\hline 2 & 0.3107 \\
4 & 0.1638 \\
8 & 0.0842 \\
\hline
\end{tabular}


where $\mathrm{T}\left[{ }^{\circ} \mathrm{C}\right]$ is the local temperature and $\mathrm{a}, \mathrm{b}$ and $\mathrm{n}$ are parameters that are constant within certain temperature intervals. The mold growth potential is defined as:

$$
m=\frac{R H}{R H_{\text {crit }}}
$$

where the critical level of the relative humidity is estimated with a polynomial function derived from extensive measurements [17].

\section{BOUNDARY CONDITIONS}

The thermal boundary conditions at the roof, inlet and outlet are prescribed temperature (Dirichlet) and are given by transient tables with measured temperature levels for a $24 \mathrm{~h}$ cycle in the middle of April, see Figure 3 . The chosen 24-hour cycle was picked out from a large amount of measurements (hourly updated for 30 years) since this interval contained reasonable values to represent the whole month. The data for humidity by volume that came with this specific interval showed to be less representative for the rest of the month since the values are increasing through-out the cycle. It was therefore decided to perform the hygrothermal analysis with a mean value for April (see Figure 6) as input to achieve more general results. The inlet and outlet air is given ambient air temperature and an equivalent temperature is set at the outer roof surface. The floor of the insulation is defined with a constant temperature of $294 \mathrm{~K}$. The boundary conditions of the walls at the short sides of the insulation are given Neumann conditions with a prescribed heat flux set to zero, since this was shown to enhance convergence with very small changes in the results.

\section{NUMERICAL SETUP}

The numerical grid was built as a structured mesh in ANSYS ICEM consisting of 500000 computational cells, which was validated in a mesh independence study as explained in Section 1.

The results presented in this article are achieved with three main simulations for different inlet velocities. To capture the impact of real outdoor conditions they were all conducted as transient simulations with a fixed time step of $1 \mathrm{~s}$ and a total time of $86400 \mathrm{~s}(24 \mathrm{~h})$. A pressure-based solver was used in ANSYS Fluent along

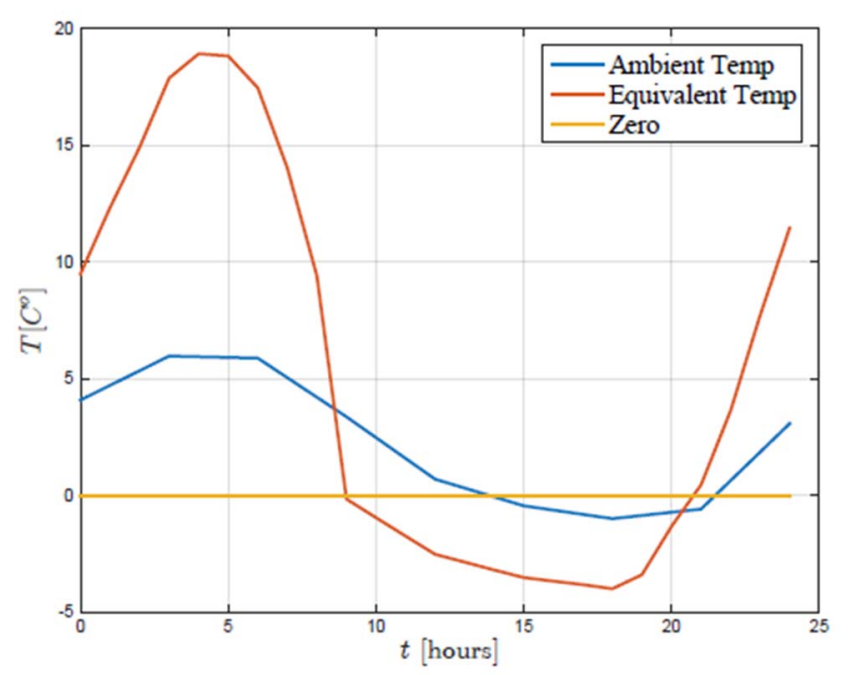

FIGURE 3. Time dependent ambient and equivalent temperatures taken from measured data plotted together with a line showing the freezing point at atmospheric pressure. 
with the implementation of the Boussinesq approximation to capture the buoyancy effects that give rise to natural convection. The numerical scheme SIMPLE was used for pressure-velocity coupling since it was proven to enhance convergence.

To model turbulence in the air space the realizable k- $\varepsilon$ model with the standard wall function was used, while the flow in the porous zone was assumed to be laminar due to low velocities. Radiation was modeled using the P1 model [15]. The permeability, $\mathrm{K}$, of the porous medium was set to $5 \cdot 10^{-8} \mathrm{~m}^{2}$, the porosity, $\phi$, to 0.332 and the thermal conductivity, $\mathrm{k}$ [W/(m.K)], of the insulation to 0.044 , assumed independent of temperature. These are values that were used when the model was validated by comparison to experimental results [13].

The simulations were initialized with constant boundary conditions for 7200 time steps and the boundary conditions for the roof and ventilation openings were then subsequently read from transient tables. Three main simulations were ran for inlet velocities of $0.0226,0.0452$ and $0.0904 \mathrm{~m} / \mathrm{s}$ corresponding to 2,4 and 8 complete air displacements per hour respectively.

Ten monitor points were created along the inner roof boundary to measure the resulting temperature at the inner roof. Each location marked in Figure 4 represents a pair of monitor points with different coordinates in direction parallel to the joists, from which the mean value was taken for analysis. The temperatures at these points were used to estimate the relative humidity and the mold growth potential along the inner roof in the post processing procedure.

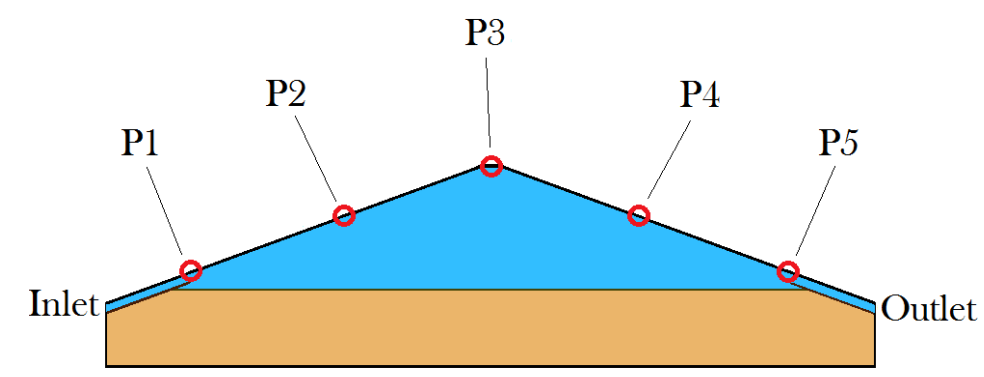

FIGURE 4. Cross section of the attic model showing the location of the 5 monitor points along the inner roof boundary. Each red mark represents two points in the direction normal to the cross section, one above the center of a joist and one in the middle between two joists.

\section{RESULTS}

The results from the transient simulations are presented in this section.

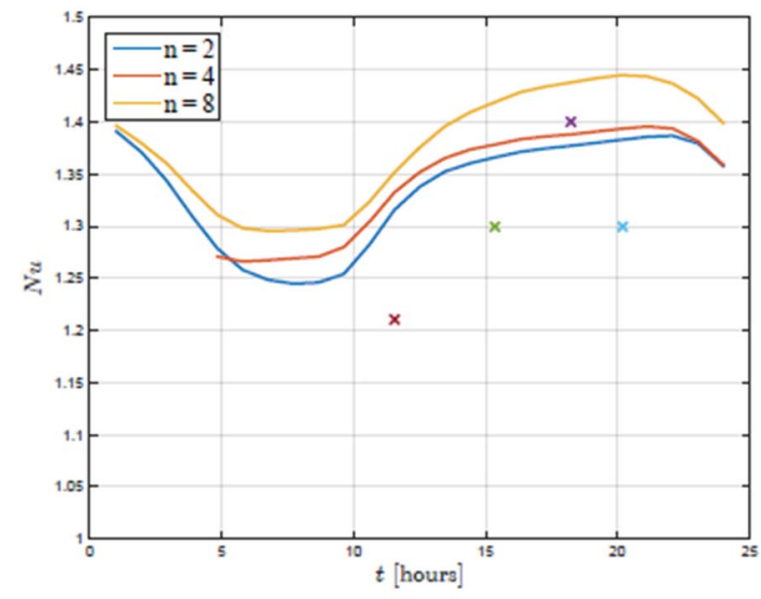

FIGURE 5. Nusselt number versus time in the insulation layer calculated each hour for all ventilation rates. $n=2, n=4$ and $n=8$ denotes 2, 4 and 8 complete air displacements per hour respectively. The four crosses show values obtained from experiments with pure natural convection [13]. 
The Nusselt number in Figure 5 was computed externally in MATLAB by importing data files with node values of temperature for the insulation volume, and

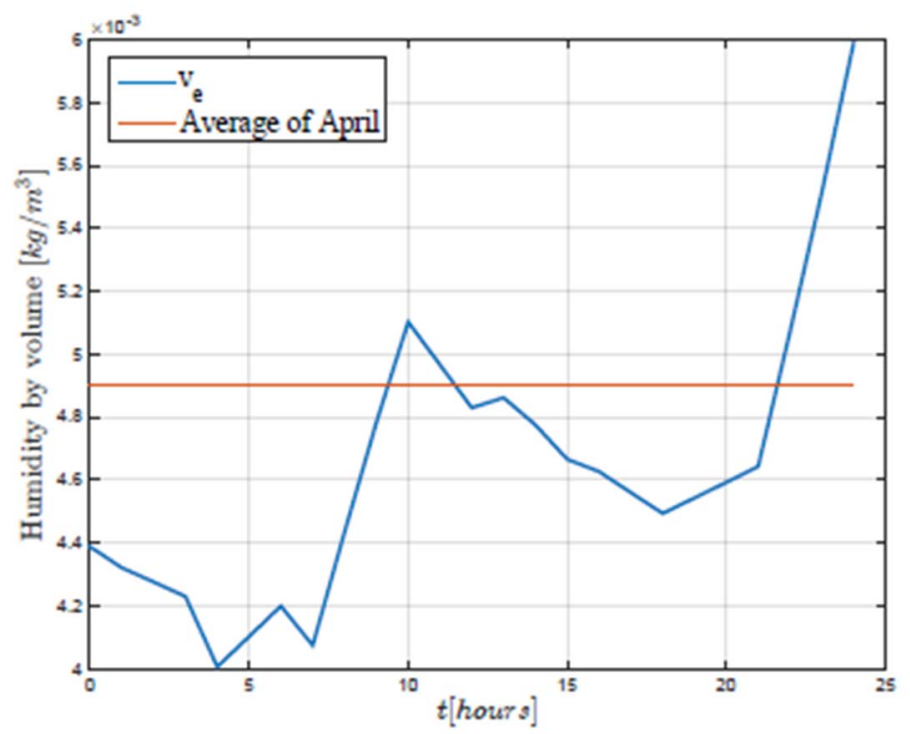

FIGURE 6. The humidity level in the outdoor air $\mathrm{v}_{\mathrm{e}}$, versus time for the chosen day plotted along with the average humidity level for April.
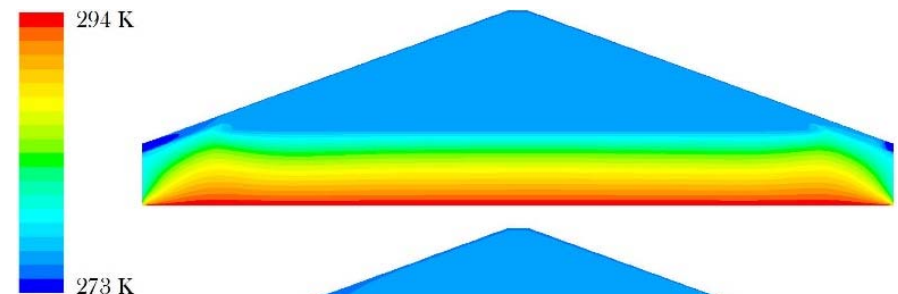

${ }_{233 \mathrm{~K}}$
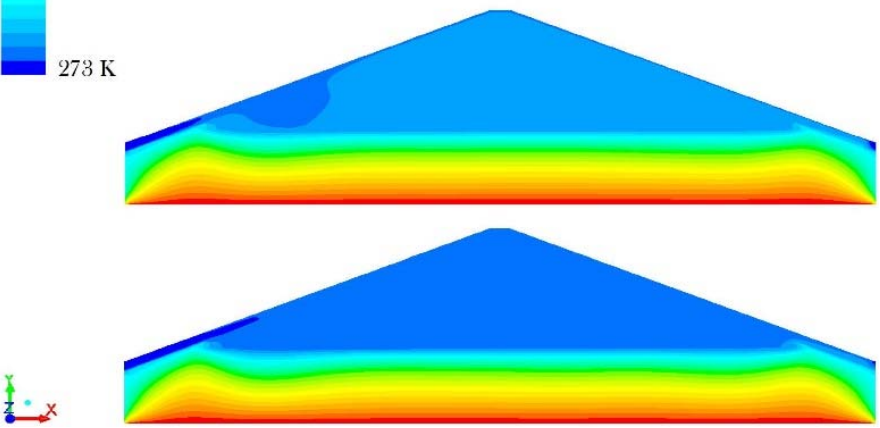

FIGURE 7. Temperature contours for the middle of the night when the temperature difference is largest, taken in the xy-plane in the middle of the attic. The plots are for, in order from above, 2, 4 and 8 complete air displacements per hour.

calculating the average fraction of the heat flux with and without convection at the heating surface, i.e. the lower surface of the attic floor insulation. One reference simulation was performed with the momentum and turbulence equations disabled in order to achieve values for the heat flux without the presence of convection, rep resenting the denominator of Equation 2, and the three cases for different air flows yielded input for the numerator. 


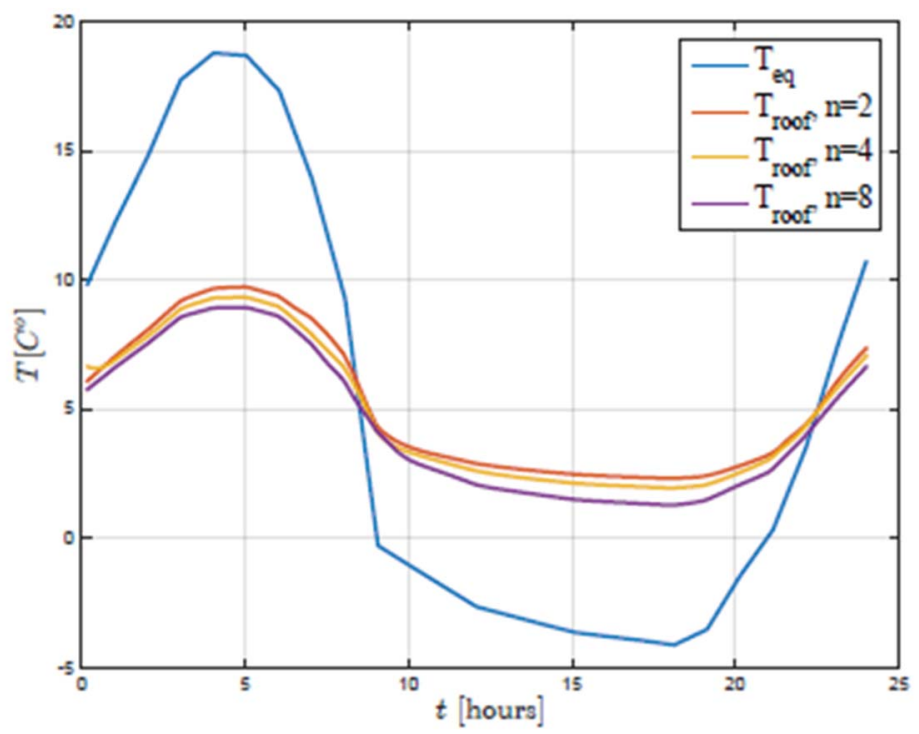

FIGURE 8. The boundary condition for the roof boundary, $\mathrm{T}_{\mathrm{eq}}$, versus time, plotted along with the mean temperature of the inner roof for the three ventilation rates.
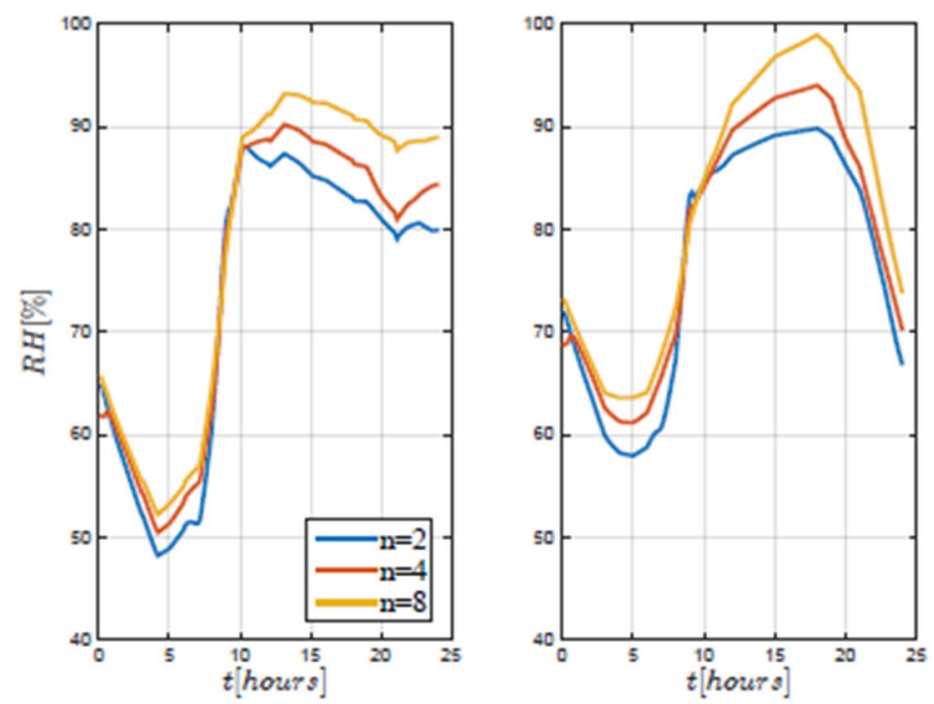

FIGURE 9. Relative humidity versus time measured at location P1 (see Figure 4). The left plot shows the values calculated from the time dependent $v_{e}$ and the right plot shows values from the averaged one, and the same goes for Figure 10, 11, 12 and 13. 

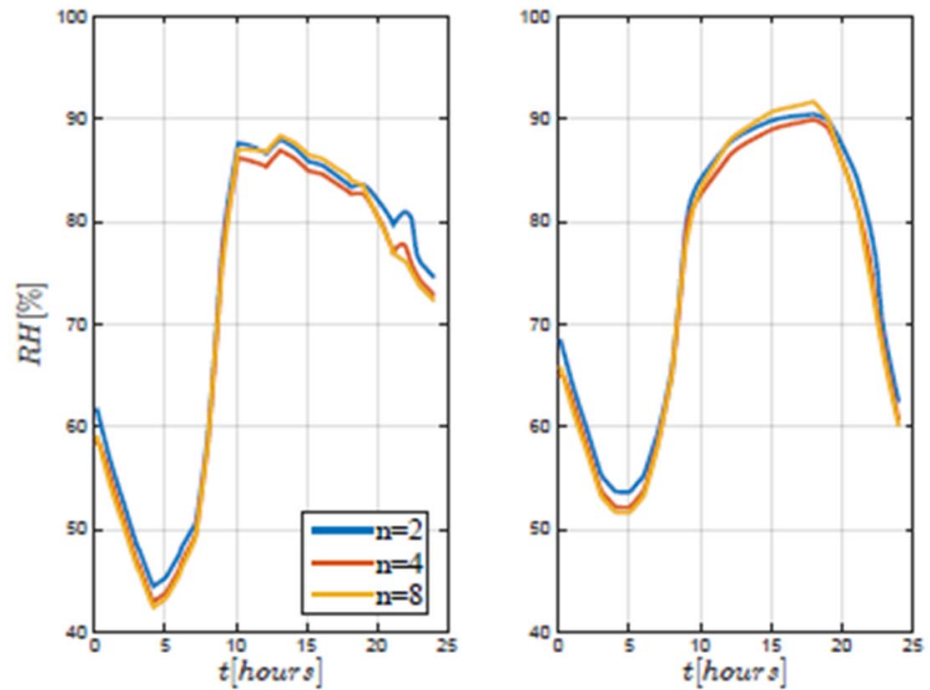

FIGURE 10. Relative humidity versus time measured at location P2.
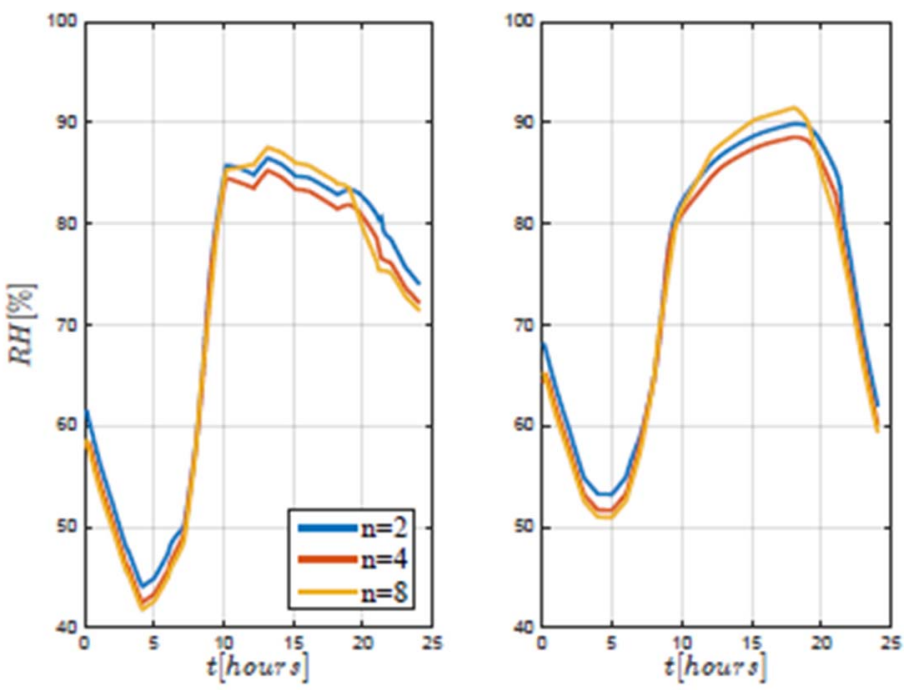

FIGURE 11. Relative humidity versus time measured at location P3.

The temperature contours in Figure 7 give understanding about the temperature distribution in the domain for different ventilation rates at the coldest point of the cycle. In Figure 7 it is seen that as the ventilation rate increases, a cloud of cold air spreads along the roof starting at the inlet. The same is proven by Figure 8 where the average temperature at the inner surface of the roof is plotted for different ventilation rates.

The levels of relative humidity and the mold growth potential are presented in two separate plots in Figure 9-18. The left plots in each figure show values 

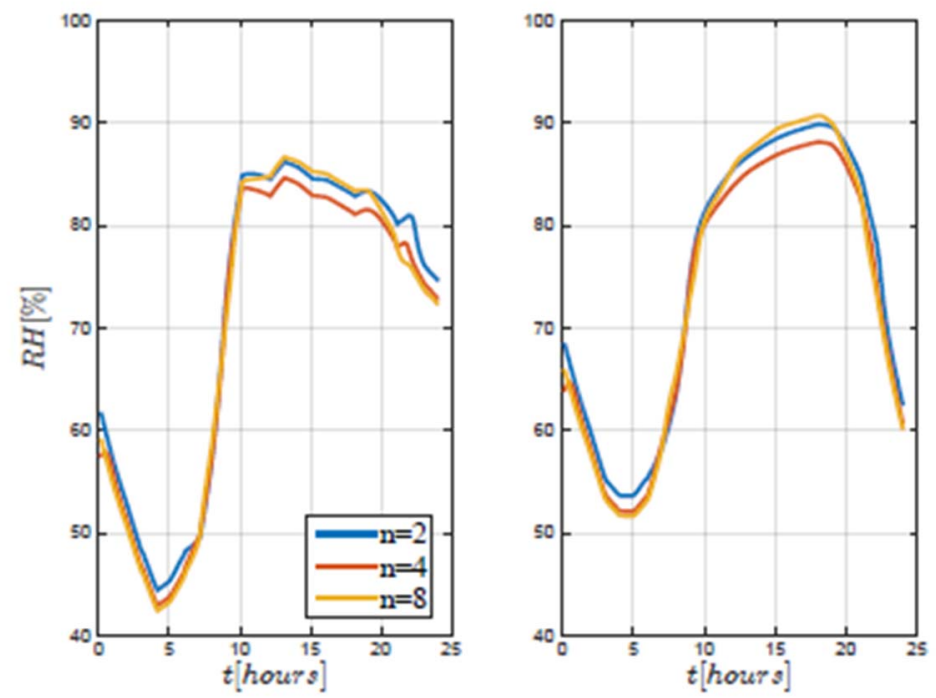

FIGURE 12. Relative humidity versus time measured at location P4.
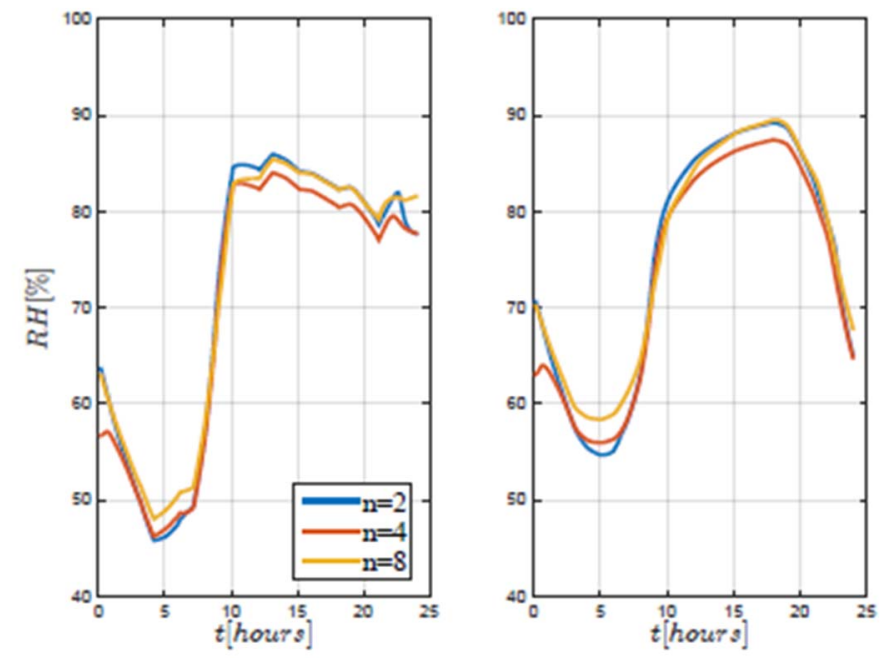

FIGURE 13. Relative humidity versus time measured at location P5.

calculated from the actual measured moisture content of the ventilated air, and the plots on the right side corresponds to the average humidity by volume for the whole month of April, as shown in Figure 6.

\section{DISCUSSION}

As seen in Figure 5 and 8, the temperature difference between the inner roof sur-face and the bottom of the attic floor insulation has a large impact on the convective heat transfer in the insulation. For the chosen cycle the conditions are such that convective heat transfer is always present in the porous medium but with varying magnitude. It is also concluded that the ventilation rate magnifies the convective transport of heat in the insulation layer, leading to a greater heat flux from the liv-ing area as the ventilation rate is increased. By studying the Nusselt number, we 

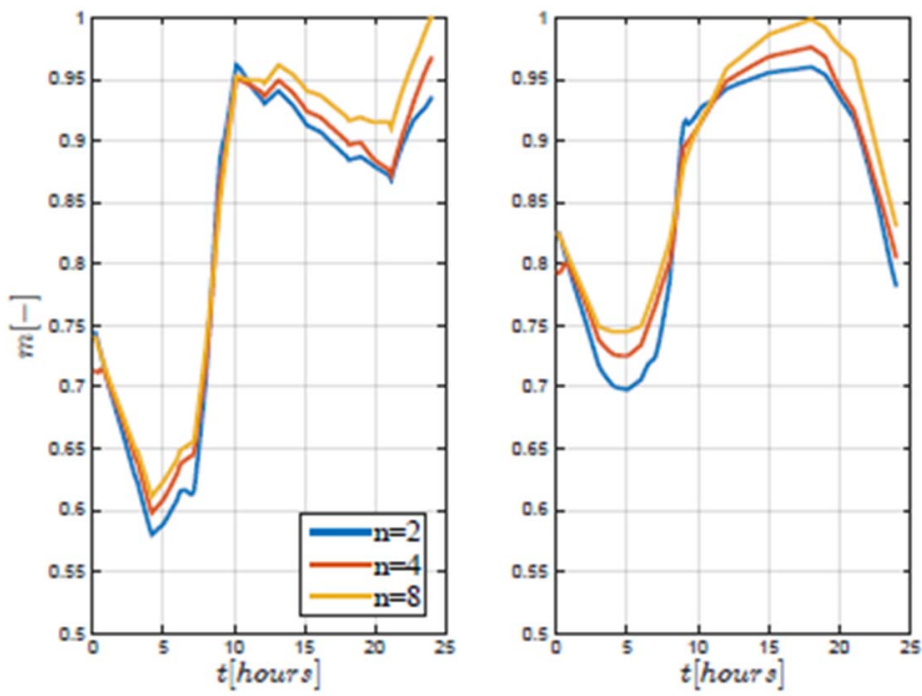

FIGURE 14. Mold growth potential, m, versus time measured at location P1 (see FIGURE 4). The left plot shows the values calculated from the time dependent $v_{e}$ and the right plot shows values from the averaged one, and the same goes for FIGURE 15, 16,17 and 18 .
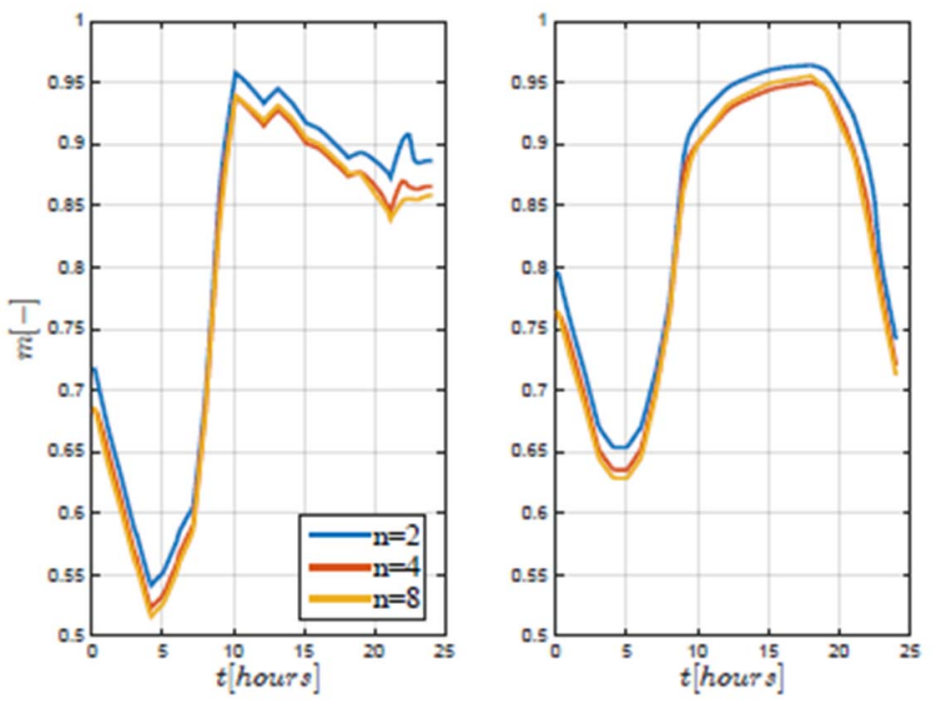

FIGURE 15. Mold growth potential, m, versus time measured at location P2. 

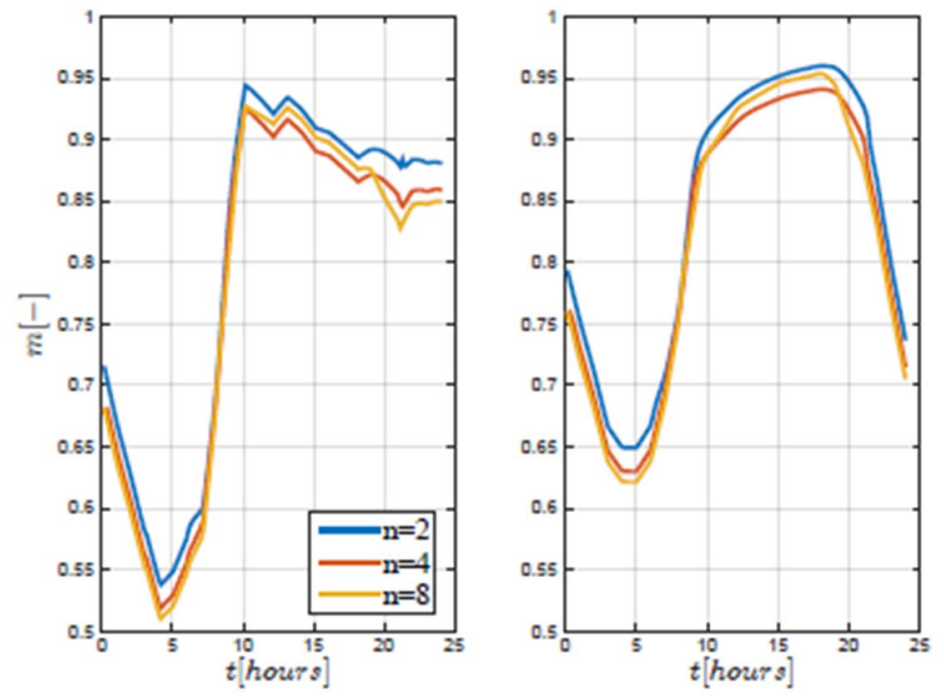

FIGURE 16. Mold growth potential, m, versus time measured at location P3.
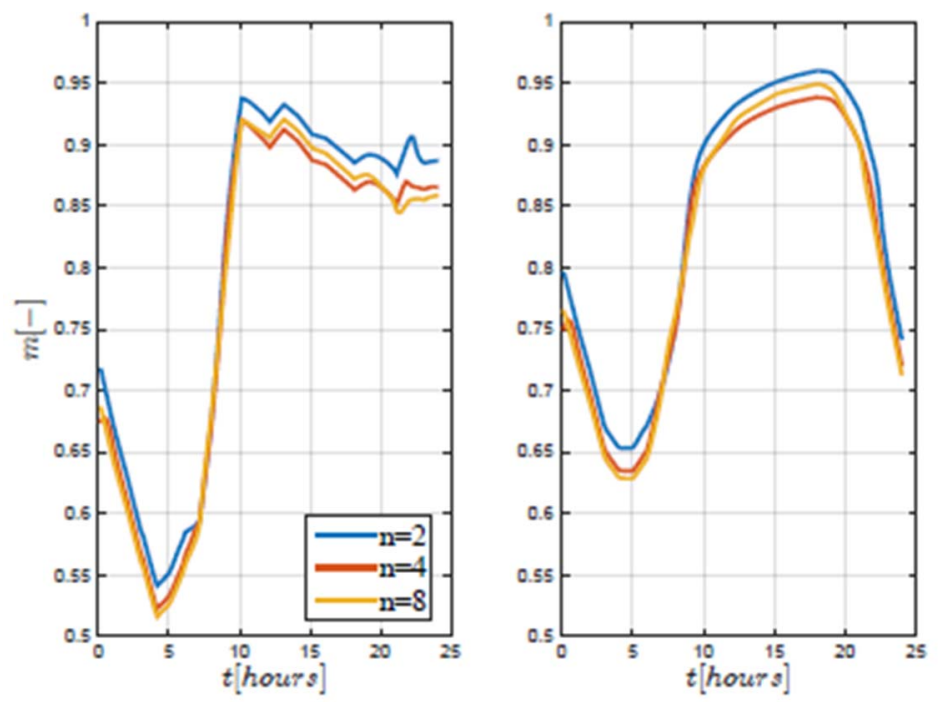

FIGURE 17. Mold growth potential, m, versus time measured at location P4.

are provided with information about the possible draw backs in terms of energy efficiency when optimizing the ventilation rate for minimal mold growth, since higher ventilation rates has a negative impact in the energy efficiency of the building. The four points in Figure 5 are values of the Nusslet number from experiments in a previous study [13]. The temperature difference over the insulation was used to determine the modified Rayleigh number according to the results in the experiments and the Nusselt numbers for those Rayleigh numbers were then added to the plot as a validation. These experiments were performed for pure natural convection which is a reason why the values for the Nusselt number from that study are slightly lower. Since there are currently no experimental results from measurements with the climatic conditions investigated in this study, the validity of the CFD model relies on the validation performed in [10]

Figure 5 shows that the Nusselt number exceeds 1 for all air displacements proving that convective heat transfer takes place in the porous medium. From Figure 5 it can be seen, glancing at Figure 3, that as the temperature difference between the roof and the floor increases, the magnitude of heat transfer due to convection also increases in the insulation. It is also noticed that the convective heat transfer gets slightly more dominant for higher ventilation rates. 

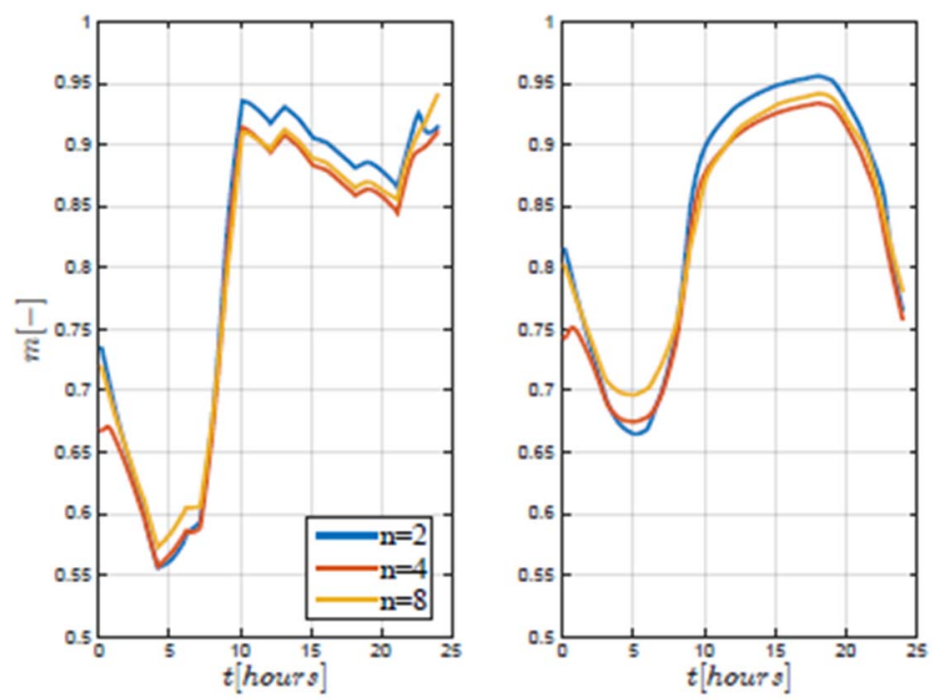

FIGURE 18. Mold growth potential, m, versus time measured at location P5.

As mentioned in Section 6, the low temperature of the ventilated air is spreading from the inlet through the attic, while in the air cavity the air is heated by the rising heat from below. For low ventilation rates $(\mathrm{n}=2)$ the air is heated up to a slightly higher temperature before it reaches the air cavity, and as the ventilation rate increases the cold air is able to travel a longer distance before it is heated up. In the contour in the middle of Figure 7 (4 complete air displacements per hour), the cold region has just reached the air cavity and a cloud of cold air is visible near the lower part of the left roof boundary, and for 8 complete air displacements per hour the cloud has spread throughout the air cavity. This tells us that the part of the roof closest to the inlet suffers the greatest risk for mold related problems.

The same can be seen by analyzing the levels of relative humidity and mold growth potential in Figure 9 to Figure 18. It is concluded that the part of the roof close to the inlet (P1 in Figure 4) is exposed to the greatest risk for mold related problems among the analyzed locations.

Finally, it should be understood that these results are taken for one specific day of the year and they are applied to one out of many attic designs, which makes these results very specific for the studied model.

\section{CONCLUSION}

The simulations yield reasonable results for the studied case. The simulations show that the heat flux in the insulation is affected by the ventilation rate leading to a slightly less energy efficient building for higher ventilation rates due to the convective heat transfer. Going from 2 to 8 complete air displacements per hour increases the heat flux through the floor of the attic by approximately $5 \%$. The obtained values of the Nusselt number are in line with previous experiments.

The relative humidity and the mold growth potential is highest at locations close to the inlet when the ambient temperature is low. At location P1 by the inlet, the highest mold growth potential $(\sim 1)$ is found for the highest ventilation rates, while the lowest ventilation rate yields the highest risk for mold growth for the rest of the locations.

\section{FUTURE WORK}

When this model was validated by comparison with experiments it was concluded that the total heat transfer in the porous medium is over predicted by Fluent, due to the inability of the code to account for resistance to radiative heat transfer in the porous zone. An investigation regarding alternative solutions for this problem is needed to achieve better accuracy of the results.

It is also of great interest to apply the tools developed in this research project on other attic designs and different inlet-outlet-configurations to optimize the ventilation strategies for minimal mold growth. 


\section{ACKNOWLEDGMENT}

This research work has been conducted with the help of financial and computer resources obtained from Swedish Energy Agency, Luleå University of Technology and the Division of Building Technology, Chalmers University of Technology.

\section{REFERENCES}

1. Jönsson, B. et al., Så mår våra hus, 2009.

2. Hagentoft, C.E. \& Kalagasidis, A.S., Mold growth control in cold attics through adaptive ventilation. validation by field measurements. Proceedings of the XI International Conference on the Performance of the Exterior Envelopes of Whole Buildings, 2010.

3. Delmas, A. \& Wilkes, K., Numerical Analysis of Heat Transfer by Conduction and Natural Convection in Loose-Fill Fiber Glass Insulation Effects of Convection on Thermal Insulation. ORNL/CON-338.

4. Wahlgren, P., Convection in Loose-fill Attic Insulation. PhD Thesis, Chalmers University of Technology, Göteborg, 2001.

5. Wahlgren, P., Measurements and simulations of natural and forced convection in loose-fill attic insulation. Journal of Thermal Envelope and Building Science, 26, pp. 93-109, 2002.

6. Shankar, V. \& Hagentoft, C.E., Influence of natural convection on the thermal properties of insulating porous medium with air cavity. Indoor air 99, Edinburgh.

7. Shankar, V. \& Hagentoft, C.E., Numerical investigation of natural convection in Horizontal porous media heated from below Comparisons with experiments. ASME International, Pittsburgh.

8. Shankar, V., Davidson, L. \& Olsson, E., Numerical Investigation of Turbulent Plumes in both Ambient and Stratified Surroundings. Journal of INDOOR AIR, Denmark.

9. Shankar, V., Davidson, L. \& Olsson, E., Ventilation by Displacement: Calculation of Flow in Vertical Plumes. ROOM VENT, Aalborg, Denmark.

10. V. Shankar, B., A \& Fransson, V., Influence of Heat Transfer Processes in Porous Media with Air Cavity - A CFD Analysis. Submitted to the conference "6th International Conference on Energy Research and Development", March 14-16, 2016, State of Kuwait.

11. V. Shankar, B., A \& Fransson, V., Numerical Analysis of the Influence of Natural Convection in Attics - A CFD Analysis. Submitted to the conference "6th International Conference on Energy Research and Development", March 14-16, 2016, State of Kuwait.

12. V. Shankar, B., A \& Fransson, V., CFD Analysis of Heat Transfer in Ventilated Attics. Submitted to the conference "IAQ 2016 Defining Indoor Air Quality: Policy, Standards and Best Practices", Sept 12 14, 2016, Alexan-dria, VA.

13. Serkitjis, M., Natural convection heat transfer in a horizontal thermal insulation layer underlying an air layer. $\mathrm{PhD}$ Thesis, Chalmers University of Technology, Göteborg, 1995.

14. Andersson, B. \& et al, Computational Fluid Dynamics for Engineers. Cam-bridge University Press: Cambridge, 2012.

15. ANSYS, Inc, ANSYS FLUENT Theory Guide. ANSYS, Inc, Canonsburg, latest edition.

16. Nield, D. \& Bejan, A., Convection in Porous Media. Springer: New York, 2013.

17. T. Ojanen, R.P., H. Viitanen, Modelling of Mold Growth. VTT - Technical Research Centre of Finland, 2007. 